\title{
Comparación ontogénica de la frecuencia de muda en Rhinella marina (Anura, Bufonidae)
}

\author{
Teófila M. Triana, Liliana M. Henao \& Manuel H. Bernal
}

Grupo de Herpetología, Eco-fisiología \& Etología, Universidad del Tolima. Altos de Santa Helena. Apartado Aéreo 546, Ibagué, Tolima, Colombia. (teofila.maria.triana@gmail.com; lmhenaom@ut.edu.co; mhbernal@ut.edu.co)

\begin{abstract}
RESUMEN. La muda es un proceso de renovación de la capa externa de la epidermis (estrato corneo) de los anfibios, la cual suministra protección contra daños, patógenos y pérdida de agua. Este trabajo evalúa la frecuencia de muda entre juveniles y adultos de Rhinella marina (Linnaeus, 1758) y la tasa de ocurrencia entre el día y la noche. Para esto, se realizaron dos observaciones diarias ( 7 am y 7 pm), entre Octubre de 2011 y Marzo de 2012, a tres grupos de individuos clasificados según su tamaño longitud rostro-cloaca, como adultos (promedio=80 mm), juveniles medianos (promedio $=19 \mathrm{~mm}$ ) y juveniles pequeños (promedio $=13 \mathrm{~mm}$ ). Estos animales fueron colocados en terrarios en el laboratorio y marcados en el dorso a través de un punto hecho con un corrector de tinta. La muda se determinó por la pérdida total de la marca y una coloración brillante en el dorso. Se encontró una diferencia significativa (Kruskal-Wallis, $\mathrm{H}=19.84$, $\mathrm{p}<0.0001$ ) en el periodo de muda entre los tres grupos de estudio: adultos $=7.5$ días, juveniles medianos $=5.4$ días, juveniles pequeños $=5.3$ días. También, en la frecuencia de animales que mudaron en el día y la noche (Ji-cuadrado, $\left.\chi^{2}=7.891, p=0.019\right)$, particularmente en los dos grupos de juveniles, quienes lo hicieron en la noche, ya que en los adultos no hubo una diferencia clara. Posiblemente, la mayor frecuencia de la muda en los juveniles puede relacionarse con su condición ontogénica, de un menor tamaño corporal, alta tasa metabólica y mayor tasa de desarrollo.
\end{abstract}

PALABRAS-CLAVE. Cambio de piel, anuros, sapo de la caña, adultos, juveniles.

ABSTRACT. Ontogenetic comparison of the molting frequency in Rhinella marina (Anura: Bufonidae). Molting is a process of constan renewal of the outer layer of epidermis (stratum corneum) in amphibians, which provides a barrier against injury, pathogens and evaporative water loss. This paper evaluates the molting frequency between juveniles and adults of Rhinella marina (Linnaeus, 1758) and between the night and day time. Two daily observations, at 7 am and 7 pm, were made between October 2011 and March 2012 to three groups of individuals, classified by their size, snout vent length, as adults (mean= $80 \mathrm{~mm}$ ), medium juveniles (mean=19 $\mathrm{mm}$ ) and small juveniles (mean=13 $\mathrm{mm}$ ). The animals were placed in terrariums in the laboratory and marked on their back with a spot of correction fluid. The molting was determined by the total loss of the mark and a bright color on the dorsal skin. We found a significant difference (Kruskal-Wallis, $H=19.84, p<0.0001$ ) in the molting frequency among the three groups: adults $=7.5$ days, medium juveniles $=5.4$ days, and small juveniles $=5.3$ days. Also, between the number of molting frogs during the night and day hours (Chi-square, $\chi^{2}=7.891, \mathrm{p}=0.019$ ), particularly in the two groups of juveniles, who moulted mostly at night, as adults did not show any clear difference. It is possible that the highest molting frequency in juveniles may be related to their ontogenetic status, with a smaller size and higher metabolic and developmental rate.

KEYWORDS. Shedding, anurans, cane toad, adults, juveniles.

La muda es un proceso de renovación constante de la parte externa de la piel en los vertebrados, que particularmente en los anfibios no presenta un patrón estacional evidente (LiNG, 1972). En este grupo, la muda se determina por el desprendimiento periódico del estrato corneo (capa externa de la epidermis), que se encuentra conformado por una serie de células queratinizadas encargadas de proteger la piel contra daños físicos (WeLLS, 2007) y regular la pérdida de agua por evaporación (Duellman \& Trueb, 1986). Durante este proceso, la parte eliminada es desprendida en una sola pieza o por parches con ayuda de la boca y sus extremidades, y suele ser ingerida con el propósito de reciclar materiales para una rápida generación epidérmica (LING, 1972), como mecanismo de recuperación de sus proteínas epidérmicas (SABAGH \& CARVALHO-ESiLva, 2008), o como suplemento de vitamina D a su dieta (BUSTARD \& MADERSON, 1965). En los anfibios la muda se realiza frecuentemente y su periodicidad varía entre unos pocos días hasta algunas semanas (DAviEs \& Withers, 1993). La muda en los anfibios es activada en el sistema nervioso central, por la hipófisis y la glándula tiroides. Sin embargo, esta activación no tiene que ser simultánea, ya que se han evidenciado mudas en individuos metamórficos con trasplantes de hipófisis en especies como Amblystoma sp. (JoRGENSEN \& LARSEN, 1960). La frecuencia de la muda, por su parte, está determinada principalmente por los niveles de actividad de la glándula tiroides (Jorgensen \& LARSEN, 1960).

BUDTZ \& LARSEN (1973), establecieron algunas fases en el proceso de muda para Bufo bufo (Linnaeus, 1758), de acuerdo a cambios histo-químicos e histológicos en la epidermis de los individuos observados, así: inter-muda, fase preparatoria, fase temprana de muda, fase tardía de muda y fase de diferenciación. De igual manera, BALAKRISHNA \& SHAKUNTALA (1988) realizaron la caracterización de la muda en Bufo melanostictus (Schneider, 1799) y establecieron cinco fases basados en cambios en el comportamiento y la coloración de los individuos: post-muda, inter-muda, pre-muda temprana, pre-muda tardía y muda. Al estudiar el comportamiento durante la muda del género Phyllomedusa (Wagler, 1830) se encontró un patrón similar al descrito por BUDTZ \& LARSEN (1973), por lo que el comportamiento de los anuros al mudar es relativamente similar, aunque su periodo de duración es diferente (CASTANHO \& De LUCA, 2001).

La frecuencia de la muda es afectada por cambios en la tasa metabólica y alteraciones en el funcionamiento de la tiroides (Jorgensen \& LARSEN, 1960). Además, 
algunas condiciones ambientales como la temperatura inciden en la frecuencia de la muda, como se ha sido reportado por Stefano \& Donoso (1964) y Meyer et al. (2012). También, varía de acuerdo con la especie y entre adultos y juveniles; por ejemplo, se han reportado periodos de muda en adultos de $B$. melanostictus de 5 a 6 días (BALAKRISHNa \& SHAKUNTALA, 1988), en B. marinus de 7 a 8 días (SCHARRER, 1958), y en B. bufo entre 7 y 11 días mientras que sus juveniles mudaron entre 4 y 7 días (LARSEN, 1976). No obstante estos datos, los reportes sobre la frecuencia de la muda entre especies y diferentes estadíos ontogénicos son escasos. Este trabajo evalúa la frecuencia de la muda entre tres grupos de la especie Rhinella marina (Linnaeus, 1758) con diferencias en su desarrollo ontogénico y en el tamaño corporal. También, compara el número de mudas registradas entre el día y la noche. De acuerdo con nuestro conocimiento, sólo en especies de Phyllomedusa, e.g. Phyllomedusa distincta (Lutz, 1950) se ha reportado que mudan al inicio de su actividad nocturna, ya que durante el día se encuentran en un estado de torpor (CASTANHO \& De LuCA, 2001).

\section{MATERIALES Y MÉTODOS}

Para el presente estudio se recolectaron 18 individuos juveniles de Rhinella marina, en COLOMBIA, Tolima: Municipio de San Luis, (Payandé, $4^{\circ} 17^{\prime} \mathrm{N}-75^{\circ} 05^{\prime} \mathrm{W}, 630 \mathrm{~m}$ ), y 11 adultos en el Tolima: Municipio de Coello (Potrerillo, $04^{\circ} 15^{\prime} \mathrm{N}$ $74^{\circ} 59^{\prime} \mathrm{W}, 430 \mathrm{~m}$ ), en dos periodos de experimentación entre los meses de Octubre de 2011 y Marzo de 2012. Estos individuos fueron transportados al laboratorio de Herpetología de la Universidad del Tolima, en donde se clasificaron en tres grupos según la longitud rostrocloaca: juveniles pequeños (promedio $=13 \pm 0.4 \mathrm{~mm}$, $\mathrm{n}=9$ ), juveniles medianos (promedio $=19.66 \pm 0.54 \mathrm{~mm}$, $\mathrm{n}=9$ ) y adultos (promedio $=79.76 \pm 5.60 \mathrm{~mm}, \mathrm{n}=11$ ). La categorización de los adultos se basó en observaciones previas de animales vocalizando y en amplexus con tamaños corporales similares en la población de estudio, en tanto que la categoría de juveniles se asignó a los organismos postmetamórficos. El sexo de los individuos no se tuvo en cuenta como una variable a evaluar en este trabajo. Posterior a la clasificación, los animales fueron señalizados en el dorso con una pequeña marca, tal y como ha sido reportado por Bouwer et al. (1953) y MEYER et al. (2012), que para el presente caso se realizó con un corrector de tinta, el cual no demostró en ensayos preliminares ningún efecto nocivo. Además, fue fácil de identificar en los animales marcados hasta su pérdida por el desprendimiento del estrato corneo. Después, los organismos fueron puestos en terrarios de $20 \times 15$ × 25 $\mathrm{cm}$ en un área ventilada del laboratorio manteniendo condiciones similares de tierra, arena, hojarasca y humedad entre el 80 al 90\%, lograda por aspersión diaria con agua declorada. La temperatura ambiental promedio para los terrarios de los juveniles durante los experimentos fue de $23.92^{\circ} \mathrm{C} \pm 1.16$ y de $25.02^{\circ} \mathrm{C} \pm 0.58$ para el caso de los adultos. El régimen de luz y oscuridad fue de 12:12 horas, aproximadamente.

Para el registro de los datos de la muda se realizaron dos observaciones diarias, una en horas de la mañana $(7: 00 \mathrm{am})$ y otra en horas de la noche $(7: 00$ $\mathrm{pm})$. Se tuvo en cuenta la ausencia de la marca en el dorso como evidencia de la ocurrencia de la muda, al igual que la observación de una piel lisa y algo húmeda. Después de la muda, se procedió a registrar y a marcar los individuos nuevamente hasta alcanzar tres periodos de muda consecutivos para cada individuo. A todos los animales se les proporcionó alimento ad libitum durante la observación de la noche, el cual consistió principalmente de adultos de dípteros, coleópteros y ortópteros, en iguales proporciones dentro de cada tratamiento, pero el doble para el caso de los adultos en comparación con los juveniles.

Para el análisis estadístico de los datos, inicialmente se empleó la prueba de Friedman para detectar si existían o no diferencias estadísticas entre los tres periodos de mudas registrados para cada individuo. Luego, se utilizó el promedio de los tres periodos de muda de cada individuo para comparar la duración de los periodos de muda entre los tres grupos evaluados: adultos, juveniles medianos y juveniles pequeños, a través de la prueba de Kruskal-Wallis, y posteriormente la prueba de Mann-Whitney para las comparaciones entre dos grupos. Finalmente, se empleó la prueba de Ji-cuadrado $\left(\chi^{2}\right)$ para determinar la asociación entre el número de mudas y su ocurrencia en el día o la noche en los tres grupos de estudio. Para establecer diferencias estadísticas entre los grupos comparados se empleó un nivel de significancia de $\mathrm{p}<0.05$.

\section{RESULTADOS}

No se encontraron diferencias significativas entre los tres periodos de muda registrados para cada individuo dentro de los grupos: adultos (Friedman, $p=0.0648$ ); juveniles pequeños (Friedman, $p=0.8799$ ); y juveniles medianos (Friedman, $\mathrm{p}=0.086$ ). La duración promedio del periodo de muda en los individuos adultos fue de 7.50 días (desviación estándar, ds \pm 0.80 ), en los juveniles medianos de 5.38 días ( $\mathrm{ds} \pm 0.11$ ), y para los juveniles pequeños de 5.33 días ( $\mathrm{ds} \pm 0.11$ ). Al comparar la frecuencia de muda para los tres grupos se encontraron diferencias estadísticamente significantes (KruskalWallis, $\mathrm{p}<0.0001)$. Esta diferencia fue generada por el grupo de los adultos con respecto de los juveniles pequeños (Mann-Whitney, $\mathrm{p}=0.0001$ ) y los juveniles medianos (Mann-Whitney, $\mathrm{p}=0.0001$ ), ya que entre los grupos de juveniles pequeños y medianos no se encontró una diferencia significativa (Mann-Whitney, $\mathrm{p}=0.9422$ ) (Fig. 1).

Al comparar el número de mudas ocurridas entre el día y la noche en los tres grupos de estudio, se encontró 
una asociación significante (Ji-cuadrado, $\chi^{2}=7.891$, $\mathrm{p}=0.019$ ). Particularmente, los juveniles pequeños y medianos mudaron con mayor frecuencia en la noche que en el día, sin que se presentara una diferencia notable en los adultos (Fig. 2).

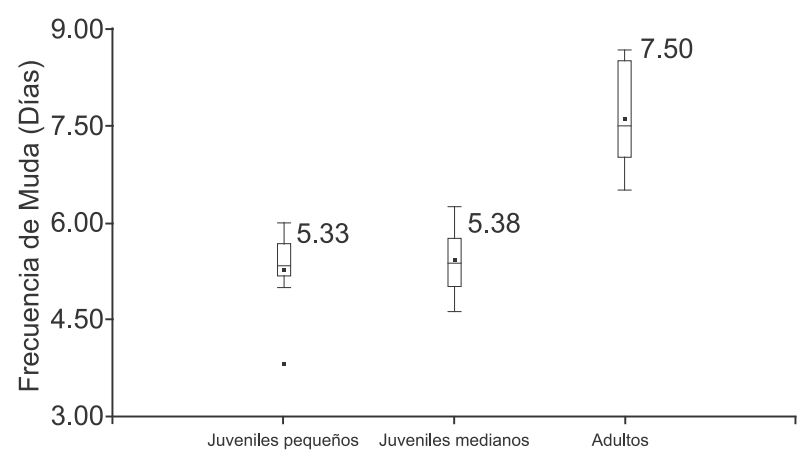

Fig. 1. Comparación del promedio de la frecuencia de muda en los tres grupos experimentales de estudio de Rhinella marina (Linnaeus, 1758). En el diagrama de cajas, el punto y el valor numérico representan la media.

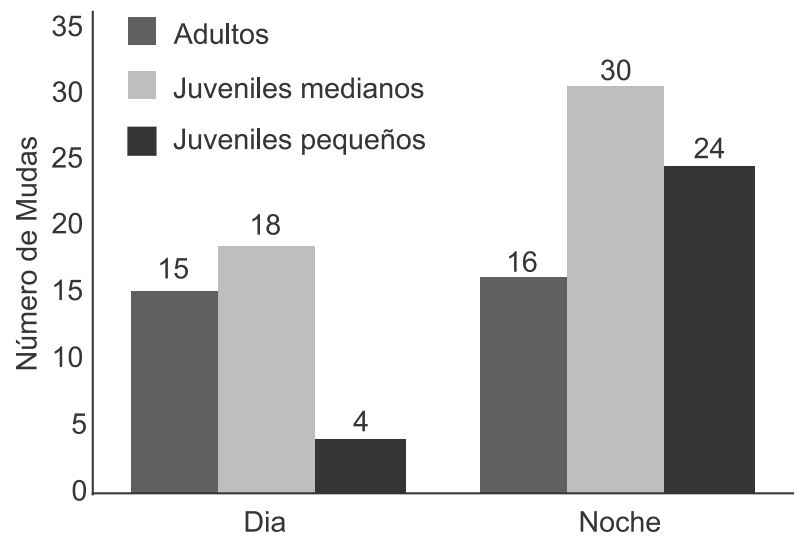

Fig. 2. Comparación del número de mudas registradas en el día y la noche en los tres grupos experimentales de estudio de Rhinella marina (Linnaeus, 1758).

\section{DISCUSIÓN}

SCHARRER (1958) realizó un estudio con adultos de Bufo marinus, y reportó una tasa de muda de 7 a 8 días a una temperatura de $25^{\circ} \mathrm{C}$. Este resultado es similar al encontrado en este trabajo para el grupo adultos (promedio de 7.5 días), el cual también se realizó a una temperatura ambiental de $25^{\circ} \mathrm{C}$. Por su parte, LARSEN (1976) reportó una frecuencia de muda para los adultos de $B$. bufo entre 7 y 11 días y para los juveniles entre 4 y 7 días a $20^{\circ} \mathrm{C}$. Estos datos, junto con los resultados del presente estudio, muestran que los juveniles tienen una mayor frecuencia de muda que los adultos. Una de las posibles explicaciones puede estar relacionada con el acelerado desarrollo y crecimiento de los juveniles (Duellman \& Trueb, 1986), los mayores requerimientos metabólicos (WeLLs, 2007; YADAV, 2008), y la necesidad de mantener una piel renovada continuamente que les hace más eficiente su proceso de respiración y entrada de agua (DUELLMAN \& TRUEB, 1986).
En este trabajo se registró un mayor número de mudas en las horas de la noche con respecto de las horas diurnas, particularmente en los dos grupos de juveniles. Aunque se requieren más datos para confirmar este resultado, mudar durante la noche podría reducir la pérdida de agua por evaporación en los anuros, la cual es menor a temperaturas bajas (Herman, 1992), y en especial cuando la piel ha quedado expuesta al ambiente por la eliminación del estrato corneo (barrera protectora). Tal vez por esto, como medida de protección, los anuros secretan una sustancia lipídica durante la muda (Herman, 1992; Castanho \& De Luca, 2001; Wells, 2007). En los juveniles fue más notorio mudar en la noche (Fig. 2), lo que podría relacionarse con una mayor pérdida de agua en comparación con los adultos (WeLLS, 2007). Debido a la poca información que hay sobre la frecuencia de la muda en anuros en relación con las horas del día o fotoperiodo, es necesario que se realicen más trabajos tanto en adultos como en juveniles de otras especies. También, sería interesante evaluar como factores ambientales asociados al hábitat de estos animales, que afectan la frecuencia de muda, como la temperatura, pueden incidir en enfermedades infecciosas asociadas a su piel. Por ejemplo, se ha reportado que la quitridiomicosis, una enfermedad cutánea causada por el hongo patógeno Batrachochytrium dendrobatidis, tiene una alta prevalencia en temperaturas bajas registradas a mayores altitudes (Piovia-Scott et al., 2011), y a temperaturas menores la frecuencia de muda disminuye (MEYER et al., 2012), lo que podría retardar la eliminación de los patógenos presentes en la piel de estos organismos.

Agradecimientos. Los autores agradecen a Johan Albeiro Romero y Claudia Marsela Montes el apoyo en el montaje y desarrollo de los experimentos, y al fondo de investigaciones de la Universidad del Tolima por la financiación del proyecto 490110 a través del cual se realizó esta investigación.

\section{REFERENCIAS BIBLIOGRÁFICAS}

Balakrishna, T. A. \& Shakuntala, K. 1988. The moult process in Bufo melanostictus (Schn.) (Anura; Bufonidae). Journal of the Indian Institute of Science 68:1-6.

Bouwer, S.; Ewer, D. W. \& ShIFF, C. 1953. Frequency of Molting in Anura. Nature 172:408.

Budtz, P. E. \& Larsen, L. O. 1973. Structure of the toad epidermis during the moulting cycle. I. Light microscopic observations in Bufo bufo (L.). Cell and Tissue Research 144(3):353-368.

Bustard, H. \& Maderson, P. F. A. 1965. The eating of shed epidermal material in squamate reptiles. Herpetologica 21(4):306-308.

Castanho, L. M. \& De Luca, I. M. S. 2001. Moulting behavior in leaffrogs of the genus Phyllomedusa (Anura: Hylidae). Zoologische Anzeiger 240:3-6.

Davies, M. \& Withers, P. C. 1993. Morphology and physiology of the anura. In: DAvies, M. \& Withers, P. C. eds. Fauna of Australia. Canberra, Australian Government Publication, C.S.I.R.O, p. 1-35.

Duellman, W. E. \& Trueb, L. 1986. Biology of Amphibians. New York, McGraw-Hill Book Company. 670p.

Herman, C. A. 1992. Endocrinology. In: Feder, M. \& Burggren, W. eds. Environmental Physiology of the Amphibians. Chicago, The University of Chicago, p. 40-54.

JoRGENSEN, L. B. \& LARSEN, L. O. 1960. Hormonal control of moulting in amphibians. Nature 185:244-245. 
Larsen, L. O. 1976. Physiology of Moulting. In: LofTs, B. ed. Physiology of the Amphibia. New York, Academic Press, p. 53-100.

LiNG, J. K. 1972. Adaptive functions of vertebrate molting cycles. American Zoologist 12(1):77-93.

Meyer, E. A.; Cramp, R. L.; Bernal, M. H. \& Franklin, C. E. 2012. Changes in cutaneous microbial abundance with sloughing: possible implications for infection and disease in amphibians. Diseases of aquatic organisms 101:235-242.

Piovia-Scott, J.; Pope K. L.; Lawler, S. P.; Cole, E. M. \& Foley, J. E. 2011. Factors related to the distribution and prevalence of the fungal pathogen Batrachochytrium dendrobatidis in Rana cascadae and other amphibians in the Klamath Mountains. Biological Conservation 144:2913-2921.
Sabagh, L. T. \& Carvalho-e-Silva, A. M. P. T. 2008. Feeding overlap in two sympatric species of Rhinella (Anura: Bufonidae) of the Atlantic Rain Forest. Revista Brasileira de Zoologia 25(2):247253.

SCHARrer, E. 1958. Molting cycles in the toad, Bufo marinus. The Anatomical Record 130:369.

Stefano, F. J. E. \& Donoso, A. O. 1964. Hypophyso-adrenal regulation of moulting in the toad. General and Comparative Endocrinology 4:473-480.

Wells, K. D. 2007. The Ecology and Behavior of Amphibians. Chicago, The University of Chicago.1148p.

YadaV, M. 2008. Amphibian Development. New Delhi, Discovery Publishing House. 232p. 